\title{
Understanding the Effects of Exponentially Decaying DC Currents on the Dual dq Control of Power Converters in Systems with High X/R
}

\author{
Daniel dos Santos Mota \\ Department of Electric Power Engineering \\ Norwegian University of Science and Technology \\ Trondheim, Norway \\ daniel.mota@ntnu.no
}

\author{
Elisabetta Tedeschi \\ Department of Electric Power Engineering \\ Norwegian University of Science and Technology \\ Trondheim, Norway \\ Department of Industrial Engineering \\ University of Trento \\ Trento, Italy \\ elisabetta.tedeschi@ntnu.no
}

\begin{abstract}
This paper investigates the root causes of detrimental oscillations in the dc link voltage of an energy storage system using a dual dq controller, operating at a high-voltage ac grid with high reactance-resistance ratio. Dual dq controllers are recommended in the literature for power converters operating under unbalanced, fault, or reduced voltage conditions. They employ two separated rotating reference frames, one for the positive and one for the negative sequence. The causes of the oscillations are investigated both theoretically and by time-domain computer simulations. As a result of the simulations, the performance of two dual controllers used in the industry is compared. In the presence of exponentially decaying dc currents, the filtering techniques employed by the controllers affect differently the performance of the proportional-integral regulators and disturb the feed forwarding and dq decoupling schemes. Ultimately, this results in undesirable oscillations in the dc-link voltage. This paper sheds light on how a fundamental phenomenon of three-phase ac systems can critically affect the control of power electronic converters. It provides a valuable insight into a possible root cause of oscillations in large electrical system applications with a considerable power converter penetration, such as large industrial plants striving for reducing greenhouse gas emissions.
\end{abstract}

Index Terms-power electronics, power systems, smart grids, renewable energies

\section{INTRODUCTION}

Offshore gas turbines responsible for heat and electricity generation in the oil and gas (O\&G) fields on the Barents, North and Norwegian Seas account for roughly $20 \%$ of the total greenhouse gas (GHG) emissions of Norway [1]. In electrical engineering terms, the total installed capacity of the 150+ gas turbines on the Norwegian Continental Shelf (NCS) was approximately $3 \mathrm{GW}$ with an yearly electrical

This research was funded by the Research Council of Norway through the PETROSENTER scheme, under the "Research Centre for Low-Emission Technology for Petroleum Activities on the Norwegian Continental Shelf" (LowEmission), grant number 296207.

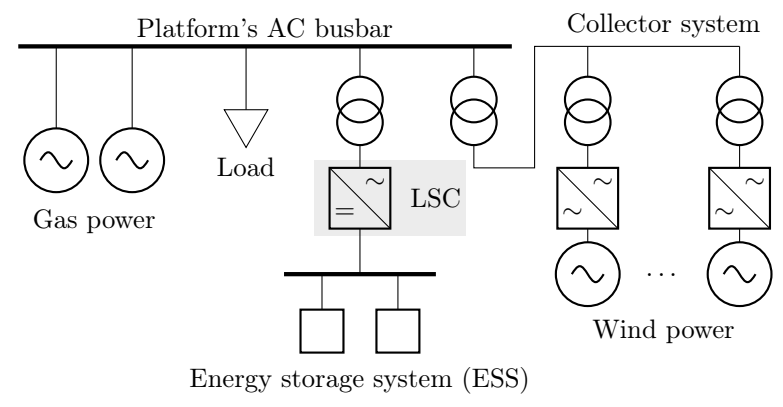

Fig. 1: Typical planned scenario for the electrical system of an $O \& G$ platform connected to an offshore wind farm.

energy consumption of 15 TWh in 2008 [2]. Although still at its infancy in a global scale, renewable offshore electricity generation is gaining momentum. Initiatives like the Hywind Tampen Project [3] point in the direction of a further increase in offshore wind electricity generation. Wind farms represent an extraordinary opportunity for reducing the offshore GHG emissions.

One typical planned scenario within this context is shown in Fig. 1. It illustrates a case study used by [4] of an O\&G platform in the North Sea isolated from the continental electrical grid. This power intensive industrial process is fed by two $35 \mathrm{MW}$ gas-powered generators. A techno-economical evaluation [5] suggests that a $12 \mathrm{MW}$ offshore wind farm would be able to reduce the GHG emissions of this platform by $30 \%$. For mitigating the effects of wind intermittency and for allowing the turbo-generators to operate at higher efficiency ranges, an energy storage system (ESS) would be connected directly to the main busbar of the platform. This paper uses the simplified, although realistic, model [6] of the case study depicted in Fig. 1 as base. See Table B.I in Appendix B for numerical data.

The reactance-resistance ratio $(X / R)$ seen from the ac 
terminals of the ESS's line side converter (LSC) towards the platform's busbar is high, as shown later in Section IV. Sudden changes in the voltage of three-phase systems with high $X / R$ induce noticeable exponentially decaying dc currents. This phenomenon is well known among electrical engineers. However, in the authors' opinion, the time domain effects of these dc components in the control of power electronic converters are not covered in detail in the literature.

During the evaluation of different contingency scenarios for the case study depicted in Fig. 1, transient oscillations were detected in the voltage of the ESS's dc link. These oscillations were triggered by sudden changes to the busbar voltage and by sudden changes to the ESS's reactive or active power. The overall stability margin of the system was clearly reduced. The contingency scenario in question had the wind farm completely disconnected and the ESS's LSC operating with dual $d q$ controllers. Such controllers work in two different rotating reference frames (RRFs), one specifically for positive sequence currents and voltages, and one for the negative sequence content. They were proposed in the literature as robust regulators for power converters operating in unbalanced three-phase systems [7, 8]. Moreover, they can also be activated in fault-ride through conditions with severe grid voltage drops [9].

In this article, the root causes of those oscillations are investigated and described. They are directly tied to the exponentially decaying dc currents of three-phase systems and to the way dual $d q$ controllers split the positive and negative sequence measurements. Moreover, a comparison of the performance of two dual controller strategies is made via time-domain simulations. The first controller is the one proposed by [7] and later employed by authors closely linked to the industry $[10,11]$. The other controller also stems from authors closely linked to the industry $[8,12,13]$.

The theoretical insights obtained from this study can benefit not only engineers working with the integration of renewable energies into $O \& G$ platforms, but also those working with converter interfaced renewable energy sources in general, specially those related to island and country-wide electrical grids.

\section{Control in a Rotating Reference Frame}

The power converter analyzed in this paper is the ESS's LSC of Fig. 1. The control of this converter is performed on a RRF which allows the use of proportional and integral (PI) regulators [14]. This is also known as vector control in the industry. It relies on $a b c \mapsto d q$ and $d q \mapsto a b c$ transformations. A comprehensive explanation on the history of these transformations, on their different variants, and on their geometrical foundations is given by [15]. See Appendix A for a list of the transformations used throughout the paper.

Let the LSC and gas-powered generators of the platform be modeled as two ideal voltage sources interconnected by a resistive and inductive (RL) impedance. Equation (1) shows the relationship between the voltages and currents in this circuit moved to the $d q_{+}$with (A.2) and (A.3). The voltages of the converter are denoted by $v_{c}$, whereas the generator voltage is denoted by $v_{g}$. The generic term grid voltage will be employed from now on when referring to $v_{g}$. A strong coupling between the $d$ and $q$ axes is evident.

$$
\left[\begin{array}{l}
v_{g_{d}} \\
v_{g_{q}}
\end{array}\right]+R\left[\begin{array}{l}
i_{d} \\
i_{q}
\end{array}\right]+\omega L\left[\begin{array}{cc}
0 & -1 \\
1 & 0
\end{array}\right]\left[\begin{array}{l}
i_{d} \\
i_{q}
\end{array}\right]+L \frac{\mathrm{d}}{\mathrm{d} t}\left[\begin{array}{l}
i_{d} \\
i_{q}
\end{array}\right]=\left[\begin{array}{l}
v_{c_{d}} \\
v_{c_{q}}
\end{array}\right]
$$

Equation (2) shows how the current in (1) can be controlled in the $d q_{+}$frame. The influence of the non-controllable grid voltage is canceled by summing it to the output of the PI controller. This is known as feed forwarding. The decoupling of the $d q$ axes is obtained with the $\omega^{\prime} L i^{\prime}$ term. The matrix in this term is named decoupling matrix $\mathbf{M}$.

$$
\left[\begin{array}{l}
v_{c_{d}} \\
v_{c_{q}}
\end{array}\right]=\left[\begin{array}{l}
v_{c_{d \mathrm{PI}}} \\
v_{c_{q \mathrm{PI}}}
\end{array}\right]+\left[\begin{array}{l}
v_{g_{d}}^{\prime} \\
v_{g_{q}}^{\prime}
\end{array}\right]+\omega^{\prime} L\left[\begin{array}{cc}
0 & -1 \\
1 & 0
\end{array}\right]\left[\begin{array}{l}
i_{d}^{\prime} \\
i_{q}^{\prime}
\end{array}\right]
$$

Assuming the feed forwarding and decoupling work perfectly, this multiple-input multiple-output (MIMO) system becomes two independent single-input single-output (SISO) systems. The Modulus Optimum tuning technique [16] with the modifications recommended by [17] is well suited for tuning these controllers.

It is worth remarking that the currents on (1) are representations of the actual quantities in the process, whereas the currents on (2) are measurements inside the controller. The same applies to the voltages and to the angular frequency. Therefore, these measurements are denoted by $v_{g}^{\prime}, \omega^{\prime}$, and $i^{\prime}$ in (2). Attenuation and phase shifts introduced by filtering degrade the performance of the PI regulators and can, potentially, disrupt the feed forwarding and the decoupling schemes during transients.

\section{DuAl $d q$ CONTROllers}

Dual $d q$ controllers were proposed in the literature as robust regulators for power electronic converters working under unbalanced and distorted conditions [7, 8]. They operate in two independent $d q$ reference frames, a $d q_{+}$for the positive sequence and a $d q_{-}$for the negative sequence, as shown in Fig. 2. A generic positive sequence measurement in the $a b c$ frame with fixed amplitude and fixed phase lag $\varphi$ becomes dc when moved to $d q_{+}$with the transformation (A.2). Conversely, a negative sequence measurement moved to the $d q_{+}$frame becomes second harmonic. For that reason, dual controllers rely on filtering the second harmonic for removing the negative sequence content from the $d q_{+}$frame. Dually, in the $d q_{-}$frame, the positive sequence content becomes second harmonic and needs, thus, to be removed by filtering.

\section{A. Notch-Based Dual dq Controller}

In 1999, a dual control scheme was proposed by [7]. The positive sequence RRF is the aforementioned $d q_{+}$. However, the authors changed the sign of $\omega$ in the Park transformation (rotation from $\alpha \beta$ to $d q$ ) for representing the negative sequence RRF. For that reason, this frame is named $d q_{-\omega}$ in this paper. The transformation between frames uses the matrices (A.1) and (A.5) from the Appendix A. Moreover, all references to 


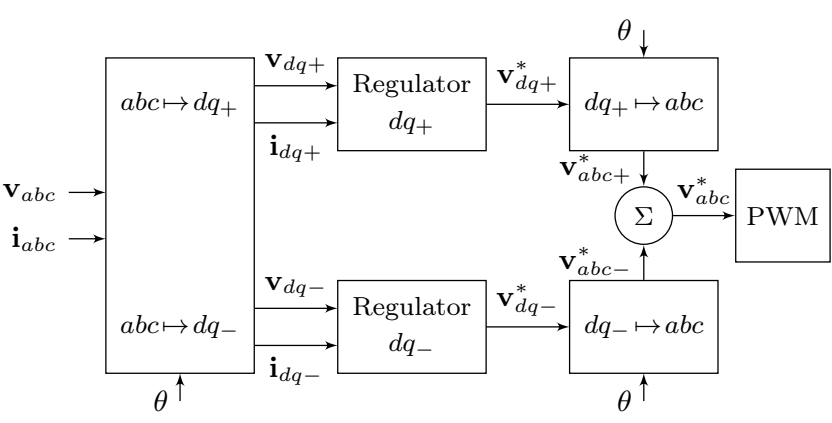

Fig. 2: Dual $d q$ controller.

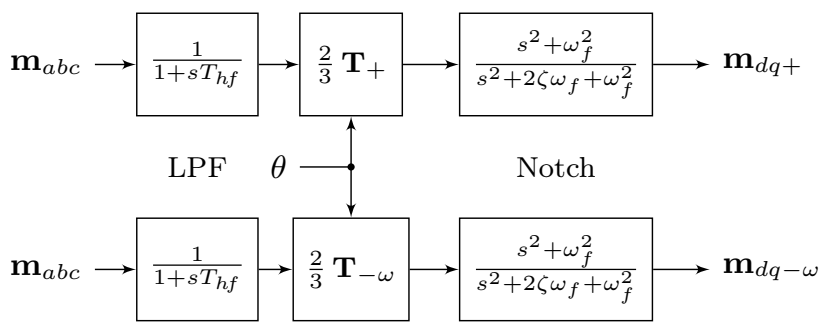

Fig. 3: Notch-filter-based transducer for the positive sequence and negative $\omega$ RRFs, adapted from $[7,9,10,18]$; the measurements $\mathbf{m}$ stand for either voltages or currents.

$d q_{-}$in Fig. 2 should be understood as $d q_{-\omega}$ for this dual controller.

A notch filter tuned to the second harmonic removes the opposite sequence measurements from each of the $d q$ frames. For this reason, this dual controller is named "notch-based" in this paper. A low-pass filter (LPF) with a cutout frequency tuned to $1000 \mathrm{~Hz}$ is added for signal conditioning at the inputs [9]. Fig. 3 shows an adaptation of the dual positive and negative $\omega$ RRF transducer used by [7], and further discussed by $[9,10,18]$. Attention must be paid when building the negative $\omega$ RRF controller as the signs of the decoupling matrix $\mathbf{M}$ of (2) are inverted in the different RRFs. This fact is illustrated on $[7,9,18]$.

The damping factor $\zeta$ in Fig. 3 defines the quality of the filter. The lower the damping, the higher the quality, i.e., lower attenuation and lower phase shifts outside the notch frequency. Nevertheless, this comes at the cost of complex conjugated poles with increasingly lower damping [19]. Computer simulations with $\zeta=\sqrt{2} / 2$ showed good results. However, for a fair comparison with the other dual controller, a unitary $\zeta$ is chosen.

\section{B. LPF-Based Dual dq Controller}

In 2012, [8] proposed a dual $d q$ controller based on [20]. The two RRFs in this controller are a positive sequence $d q_{+}$ and a negative sequence $d q_{-}$, see (A.1) and (A.4). It is worth noting that the decoupling matrix $\mathbf{M}$ of (2) can be directly used in the $d q_{-}$frame. This can be verified by using the matrices

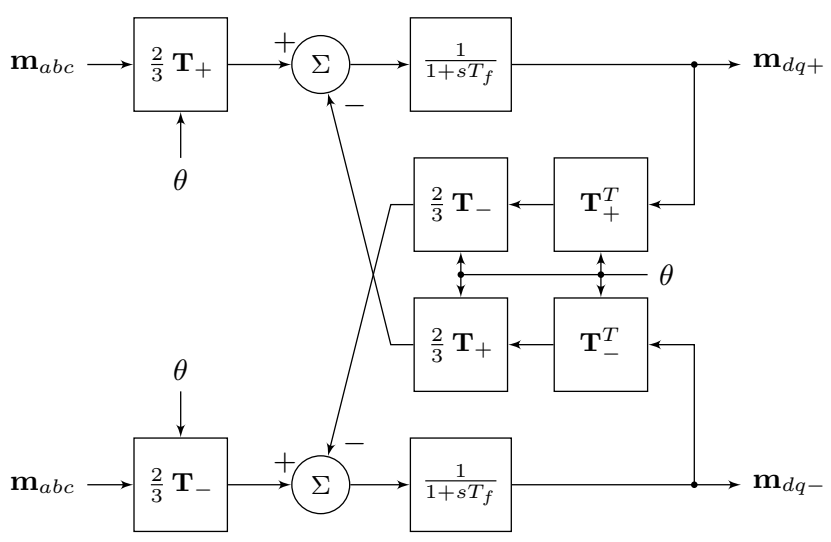

Fig. 4: LPF-based transducer for positive and negative sequence RRFs, adapted from [20]; the measurements $\mathbf{m}$ stand for either voltages or currents.

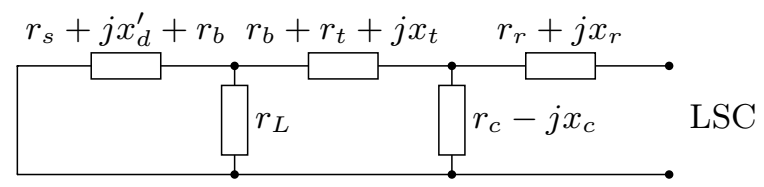

Fig. 5: Single-line diagram used for the $X / R$ calculation.

in the Appendix A to re-write the quantities of (1) into the $d q_{-}$frame.

The measurement principles of the dual controller proposed by [8] rely on LPFs as shown in Fig. 4. For that reason, the controller is denoted as "LPF-based" in this paper. The measurements $\mathbf{m}_{d q+}$ and $\mathbf{m}_{d q-}$ are the outputs of LPFs. They are transformed back to the other RRFs and become second harmonic. At the summation blocks, they cancel the undesirable opposite sequence content from each RRF.

According to [20], the cutout frequency of the LPFs should be lower or equal to $f_{n} / \sqrt{2}$, where $f_{n}$ is the fundamental, for guaranteeing a stable and damped response of the transducer. The cutout frequency is, in this paper, set to $f_{n} / \sqrt{2}$.

\section{Establishing a Representative Range of $X / R$}

It is now time to set the dual controllers within the high $X / R$ scenario depicted in Fig. 1. The reactance-resistance ratio in question in this paper is the one seen from the ac terminals of the ESS's LSC towards the platform's busbar. In this section, a representative range of values for $X / R$ is determined. Fig. 5 summarizes this calculation.

As previously mentioned, the wind farm transformer at the platform is disconnected from the busbar. The two synchronous generators are modeled as a voltage source behind the transient reactance $x_{d}^{\prime}$ in series with the stator resistance $r_{s}$. The resistance $r_{b}$ represents the losses in the circuit breaker and other high-voltage (HV) devices. The load is simplified as a fixed resistor $r_{L}$. Typical short-circuit impedance and full load losses taken from [21] are used to derive $x_{t}$ and $r_{t}$ of the ESS's transformer. Moreover, $x_{t}$ is adopted as the line-side 


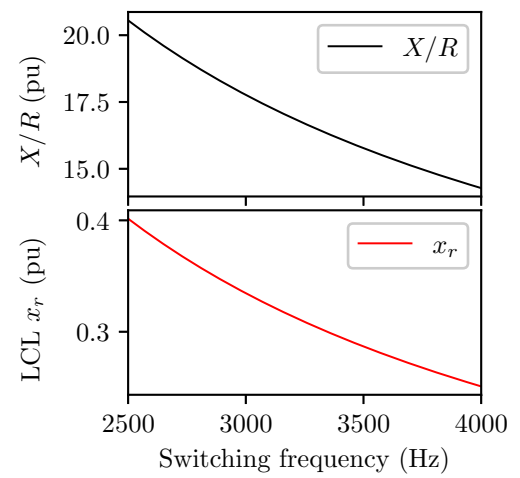

Fig. 6: Representative range of $X / R$ and LCL converter-side reactance versus PWM switching frequency for the scenario in Fig. 1 with the wind farm disconnected.

reactance of the converter's inductor-capacitor-inductor (LCL) filter. See Tables B.I and B.II in Appendix B for numerical values.

The main contributor to the $X / R$ seen from the LSC's ac terminals is the converter-side reactor of the LCL filter, $x_{r}$ in Fig. 5. For MW-sized converters like the ones in [22], paralleled two-level insulated-gate bipolar transistor (IGBT) bridges with individual LCL filters are typically employed. Without loosing generality for estimating the range of $X / R$, the LSC can be modeled as one single converter with one single LCL.

The design of LCL filters is well documented in the literature [23-26]. In this paper, the criteria described by [26] are used for calculating the reactances $x_{r}$ and $x_{c}$ and the resistance $r_{c}$ in Fig. 5. Those depend on the pulse-width modulation (PWM) switching frequency $f_{s w}$ and on the dc link's rated voltage. For MW-sized converters such as the ones in [27], $f_{s w}$ can be selected between $1 \mathrm{kHz}$ and $4 \mathrm{kHz}$. Fig. 6 shows a representative range of values for $X / R$ and for the calculated values of $x_{r}$ as a function of the PWM switching frequency.

\section{Exponentially DeCAying DC CURRENTS IN THREE-PHASE SYSTEMS}

The impedance seen from the ac terminals of the ESS's LSC in Fig. 1 has an $X / R$ on the range of 10 to 20. Sudden changes at the voltage of the LSC induce exponentially decaying dc currents with a transient time $(3 \tau)$ between $80 \mathrm{~ms}$ to $160 \mathrm{~ms}$. For understanding the effects of exponentially decaying dc currents on the control of power converters, it is important to recapitulate a few basic principles.

If a positive-sequence three-phase voltage source with amplitude $V$ is connected to a RL load, the current supplied to each phase of the load is

$$
i(t)=\frac{V}{\|Z\|}\left[-\mathrm{e}^{-t / \tau} \sin (\gamma-\varphi)+\sin (\omega t+\gamma-\varphi)\right]
$$

where $\varphi=\arctan (\omega L / R),\|Z\|=\sqrt{(\omega L)^{2}+R^{2}}$, and $\gamma$ is the initial phase of the voltage, i.e., zero, or $-2 \pi / 3$, or $+2 \pi / 3$.
The currents in (3) can be split into exponentially decaying and sinusoidal components. The latter form a positive sequence in response to the feeding voltage. The former, on the other hand, are neither positive nor negative sequence. There is no sinusoidal variation with time. They do not contribute to zero sequence either as their sum is continuously equal to zero. Equation (4) shows the three-phase exponentially decaying current components organized as a vector.

$$
\mathbf{i}_{\text {exp }}=\frac{V}{\|Z\|} \mathrm{e}^{-t / \tau}\left[\begin{array}{c}
\sin (\varphi) \\
\sin (\varphi+2 \pi / 3) \\
\sin (\varphi-2 \pi / 3)
\end{array}\right]
$$

\section{A. Positive Sequence RRF}

Equation (5) shows (4) moved into the positive sequence RRF. The exponentially decaying dc currents in the $a b c$ frame become an exponentially decaying fundamental in $d q_{+}$.

$$
\mathbf{i}_{\exp _{d q+}}(t)=\frac{2 \mathbf{T}_{+}}{3} \mathbf{i}_{\exp }(t)=\frac{V \mathrm{e}^{-t / \tau}}{\|Z\|}\left[\begin{array}{c}
-\cos (\omega t+\varphi) \\
\sin (\omega t+\varphi)
\end{array}\right]
$$

The instantaneous power supplied to a three-phase circuit is given by (6). The power dissipated by the currents in (5) can be calculated with (6), resulting in (7).

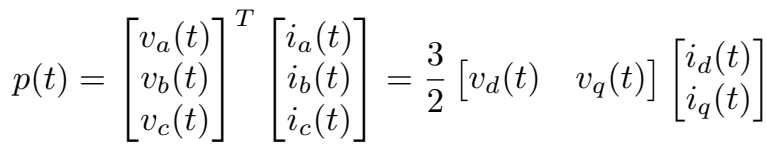

$$
\begin{aligned}
& p(t)=-\frac{3}{2} \frac{V^{2}}{\|Z\|} \mathrm{e}^{-t / \tau} \cos (\omega t+\varphi)
\end{aligned}
$$

The transient dc currents in the $a b c$ frame cause an oscillation in the instantaneous power delivered by the voltage source to the load. Active power oscillations directly affect the dc-link voltage of the converter. If a dual controller is expected to dampen exponentially decaying dc components in the $a b c$ frame, it should preserve the fundamental frequency components of the measurements in the $d q$ frame. However, for a dual controller to work, it needs to filter out the second harmonic from its $d q$ frames. Unfortunately, both tasks cannot be perfectly achieved with zero attenuation and phase shift with causal filters [18].

\section{B. Negative Sequence RRF}

The LPF-based controller uses the negative sequence $d q_{-}$ frame. Equation (8) shows (4) moved to $d q_{-}$with (A.4). The exponentially decaying dc currents in the $a b c$ frame appear as exponentially decaying fundamental in $d q_{-}$.

$$
\mathbf{i}_{\exp _{d q-}}(t)=\frac{2 \mathbf{T}_{-}}{3} \mathbf{i}_{\exp }(t)=\frac{V \mathrm{e}^{-t / \tau}}{\|Z\|}\left[\begin{array}{c}
\cos (\omega t-\varphi) \\
-\sin (\omega t-\varphi)
\end{array}\right]
$$

\section{Negative $\omega R R F$}

The notch-based dual controller uses a negative $\omega$ RRF, see Appendix A. Equation (9) shows (4) moved to the $d q_{-\omega}$ frame.

$$
\mathbf{i}_{\exp _{d q-\omega t}}(t)=\frac{2 \mathbf{T}_{-\omega} \mathbf{i}_{\exp }(t)}{3}=\frac{V \mathrm{e}^{-t / \tau}}{\|Z\|}\left[\begin{array}{l}
-\cos (\omega t-\varphi) \\
-\sin (\omega t-\varphi)
\end{array}\right]
$$


Notice that direct quantities in $d q_{-\omega}$ feature the opposite sign of the ones in the $d q_{-}$frame. The quadrature quantities, on the other hand, have the same sign. This has to be taken into consideration when comparing the two types of dual controllers.

\section{Vi. Comparing the Measurement Principles of the DUAL $d q$ CONTROLlERS}

The positive and negative sequence measurement principles of the notch-based and the LPF-based dual controllers are compared in this section. This is done by simulating the connection of a three-phase positive-sequence voltage source to an RL load. The voltage amplitude is set to $0.1 \mathrm{pu}$, the frequency to $60 \mathrm{~Hz}$, the reactance to $0.5 \mathrm{pu}$, and the resistance to $0.025 \mathrm{pu}$. The angular frequency measurement is assumed to be instantaneous. Moreover, zero delay is assumed for the measurement of $\theta$ in Fig. 2.

The currents in the $d q$ frames can be analytically determined with (3) and the transformation matrices of the Appendix A. The reactance-resistance ratio is at the highest representative range for the scenario analyzed in this paper. This highlights the effects of the exponentially decaying dc components without reducing the generality of the results. It is necessary to remark that the direct negative sequence measurements $\left(d_{-}\right)$ of the LPF-based dual controller are multiplied by -1 in all figures for allowing a direct comparison with the notch-based principle.

Fig. 7a shows the $d q_{+}$voltage measured by the notch and the LPF-based measuring principles. The response for $v_{d+}$ is similar for both principles except for the second order characteristics on the notch-based measurement. The notchbased transducer measures the expected value of $v_{q+}=0 \mathrm{pu}$. However, the cross-coupling of RRFs of the LPF-based principle (see Fig. 4) shows its influence. A deviation of almost $0.02 \mathrm{pu}$ is observed in the $v_{q+}$ measured by the LPF-based transducer after the $0.1 \mathrm{pu}$ change in the positive-sequence voltage.

Fig. 7c shows $d q_{-}$and $d q_{-\omega}$ measurements with the notch and the LPF-based measuring principles. Although featuring a sharper increase at the beginning, the measurements in both direct and quadrature axes with the notch-based principle return to zero faster than the measurements with the LPFbased transducer.

Figs. $7 \mathrm{~b}$ and $7 \mathrm{~d}$ show the measurements of the currents in the $d q_{+}, d q_{-}$, and $d q_{-\omega}$ frames. In all cases, the phase shifts featured by the LPF-based transducer are larger than the ones featured by the notch-based. This is an indication that the performance of the PI regulators, as well as the decoupling of the $d q$ axes will be worse for the LPF-based dual controller. Moreover, this also indicates that the notchbased dual controller will have a better overall performance during transients than its counterpart.

\section{Comparing the Performance of the Dual $d q$ CONTROLLERS}

In this section, the performance of the notch-based and LPF-based dual controllers employed at a scenario with high
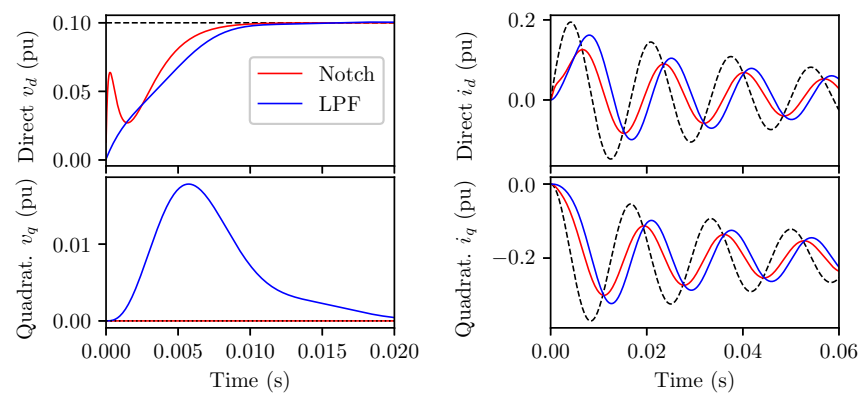

(a) Voltage $d q_{+}$

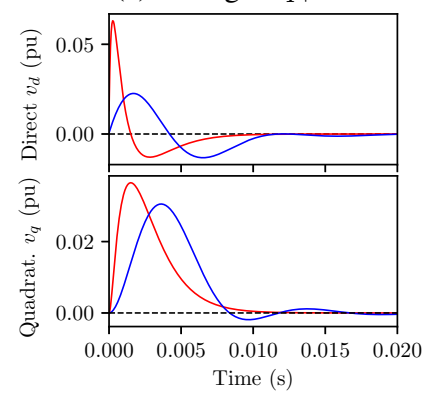

(b) Current $d q_{+}$

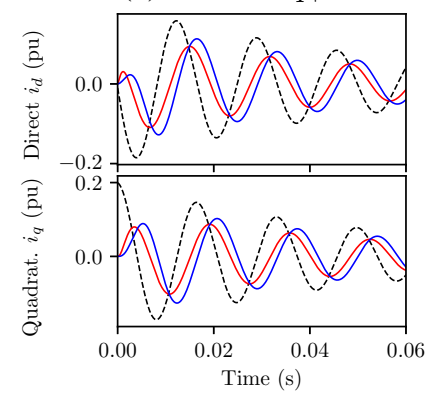

(c) Voltage $d q_{-}$and $d q_{-\omega}$

Fig. 7: Comparison of the measurement principles; notchbased in red, LPF-based in blue, ideal measurements in dashed black.

$X / R$ as the one in Fig. 1 are evaluated. This is done by simulating a controlled voltage source representing the LSC connected to the grid via a reactance in series with a resistance. Initially, the grid voltage is a pure positive sequence with $1 \mathrm{pu}$ amplitude. The reactance and resistance are the same as the ones employed in Section VI. All PI regulators have the structure $K(1+s T) /(s T)$. They are tuned equally in both dual controllers. The integrator time is adjusted to $T i=X /(\omega R)$, whereas the proportional gain $K$ is set to 4 (see Section II).

In the first two tests shown, all current references are set to zero. Therefore, deviations from zero in the currents and instantaneous power are key performance indicators. The higher the deviations and the longer their duration, the worse the performance. For the last test, however, a step is applied to the reference of the positive sequence quadrature current which results in a pure reactive current. The active power dissipated by this current is equal to $0.001 \mathrm{pu}$ and can be treated, for all practical purposes, as zero in this comparison. For the pu bases, see Table B.I.

Fig. 8a shows the response of the notch-based regulator to an instantaneous $0.2 \mathrm{pu}$ drop at the grid voltage at $t=0.5 \mathrm{~s}$. The feed forwarding of the grid voltage works quickly, resulting in almost instantaneous changes to the converter voltage. The exponentially decaying currents induced by the sudden change in the grid voltage are not easily discernible. The notch-based regulator brings the currents back to zero in less than $50 \mathrm{~ms}$. There is a small perturbation in the active power which goes over $0.01 \mathrm{pu}$ for approximately $10 \mathrm{~ms}$. For the LPF-based dual controller, Fig. 8b, the feed forwarding is 


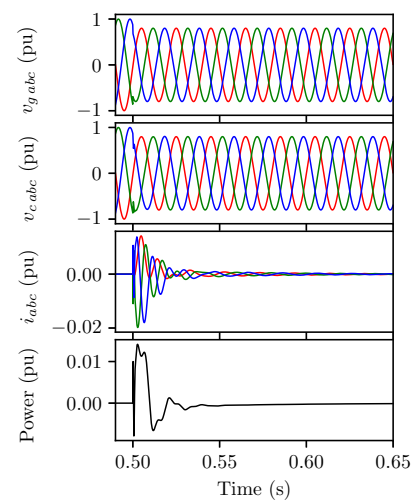

(a) Notch-based

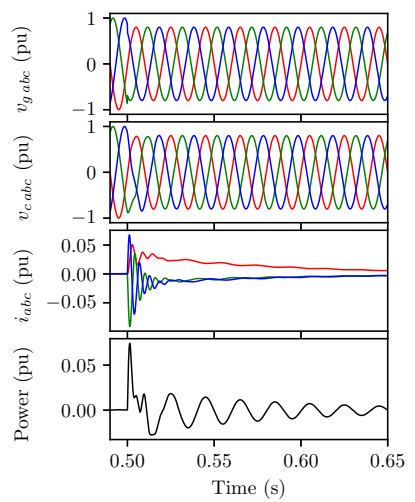

(b) LPF-based

Fig. 8: Response to a drop in the grid voltage, positive sequence only; phase $a$ in red, $b$ in green, $c$ in blue, and instantaneous power in black.

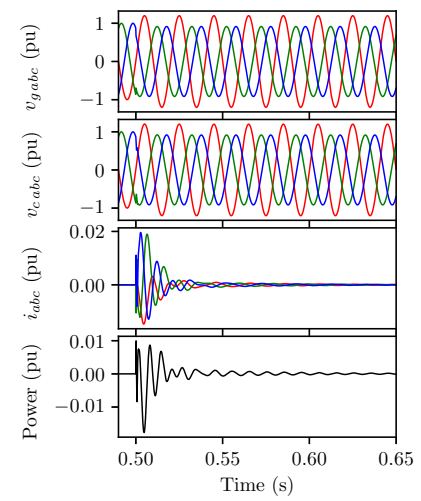

(a) Notch-based

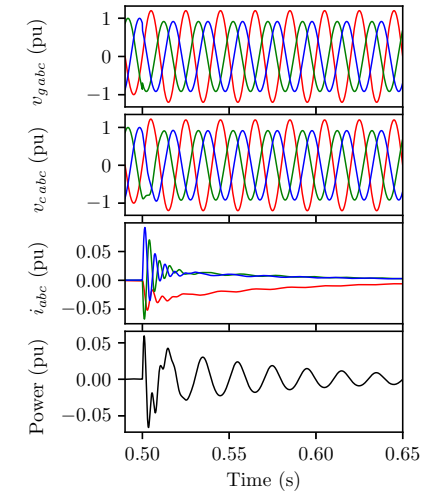

(b) LPF-based

Fig. 9: Response to a step at the grid voltage negative sequence content; phase $a$ in red, $b$ in green, $c$ in blue, and instantaneous power in black.

not as fast as for the notch-based one. The transitions in the converter voltage right after $t=0.5 \mathrm{~s}$ are smoother. Moreover, the instantaneous power for the LPF-based controller peaks at $0.074 \mathrm{pu}$ and, later, oscillates with $60 \mathrm{~Hz}$ for more than $150 \mathrm{~ms}$. This is due to the slow decay of the dc components of the current.

Fig. 9a shows the response of the notch-based regulator to a sudden introduction of a negative sequence with $0.2 \mathrm{pu}$ amplitude at $t=0.5 \mathrm{~s}$. The notch-based regulator manages to bring the currents back to zero within $50 \mathrm{~ms}$, whereas the LPF-based one does not (Fig. 9b). Similarly to the previous results with the LPF-based controller, the decay of the dc components in the current is slow causing the instantaneous power to oscillate with $60 \mathrm{~Hz}$.

Fig. 10 shows the last tests performed with the dual controllers. A step of $-0.2 \mathrm{pu}$ is applied at the positive sequence quadrature current reference at $t=0.5 \mathrm{~s}$. A remarkable difference between the converters response is the time it takes for them to achieve a balanced supply of current. The notch-

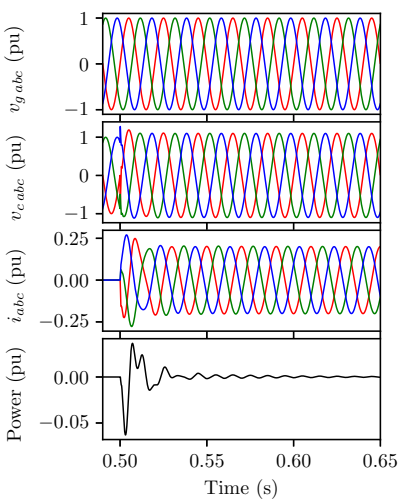

(a) Notch-based

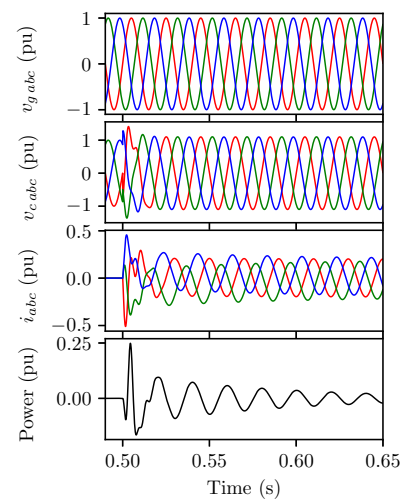

(b) LPF-based
Fig. 10: Response to step at the $i_{q+}$ reference; phase $a$ in red, $b$ in green, $c$ in blue, and instantaneous power in black.

TABLE I: Comparison of the performance of the dual controllers; the higher $\Delta p$ the worse; $1 \mathrm{pu}=9.65 \mathrm{MW}$.

\begin{tabular}{lcc}
\hline Test & $\begin{array}{c}\text { Notch-based } \\
\text { Max. } \Delta p \text { pu }\end{array}$ & $\begin{array}{c}\text { LPF-based } \\
\text { Max. } \Delta p \text { pu }\end{array}$ \\
\hline Pos. seq. grid volt. drop of $0.2 \mathrm{pu}$ & 0.014 & 0.074 \\
Neg. seq. step -0.2 pu at grid voltage & 0.018 & 0.066 \\
Step at the reference of $i_{q+}$ & 0.036 & 0.249 \\
\hline
\end{tabular}

based controller manages it within $30 \mathrm{~ms}$, whereas the LPFbased takes more than $150 \mathrm{~ms}$.

The overall performance of the notch-based regulator is better than the one of the LPF-based, see Table I. The notch-based is able to dampen the dc current components in less than the expected transient time $3 \tau=160 \mathrm{~ms}$, being the components caused by uncontrolled grid voltage changes (Figs. 8 and 9) or caused by controlled LSC voltage changes (Fig. 10). The LPF-based, on the other hand, has a much worse performance. The larger phase shifts in the measurements (Fig. 7) make the feed forwarding of the grid voltage slower and, in addition, degrade the response of the PI regulators with their decoupling scheme.

\section{CONCLUSIONS}

This paper investigated the root causes of undesired transient oscillations in the dc link of an ESS using a dual $d q$ controller in an electrical system with high $X / R$.

Dual $d q$ controllers were proposed in the literature as robust regulators for power electronic converters operating under unbalanced and distorted conditions. They work in two RRFs and rely on filtering the second harmonic from the measurements in the $d q$ frame for correctly isolating the positive from the negative sequence content.

Sudden changes to the voltage of three-phase electrical systems with high $X / R$ induce exponentially decaying dc currents in the $a b c$ frame. These currents become exponentially decaying sinusoidal signals with the fundamental frequency when moved to the $d q$ frame. They need to be properly 
measured if a controller is to dampen them. Dual $d q$ controllers are thus faced with two conflicting tasks to be performed concurrently in their $d q$ frames: 1) remove the second harmonic to split positive and negative sequences; and 2) preserve the fundamental frequency components of the measurements.

As a part of the investigations on the root causes of the oscillations in the dc link of the ESS of Fig. 1, the performance of two dual $d q$ controllers were compared. The simulations on a conceptual model presented in Section VII showed that the dual controllers are stable, albeit with different dynamic responses in spite of the identical tuning of the PI regulators. The notch-based controller handled transients considerably better than the LPF-based did. From the response to the step at the quadrature current reference, it is possible to infer that, for the same proportional gain, the LPF-based controller is less stable than the notch-based.

The filtering techniques necessary for a dual $d q$ controller to work introduce phase shifts to the measurement of the exponentially decaying currents. In addition to degrading the performance of the PI regulators, these phase shifts also delay the feed forwarding of the grid voltage and perturb the decoupling of the $d q$ axes during transients. This results in noticeable oscillations in the active power exchange between converter and grid, which, ultimately, manifest themselves as undesirable transient oscillations in the dc-link voltage.

The analysis of the problem in the time-domain, with focus on the effects of exponentially decaying dc currents in threephase systems with high $X / R$ in the dual $d q$ control of power electronic converters is the main contribution of this paper. It provides a valuable insight into a possible root cause of oscillations in high capacity power converters operating in large electrical systems, being them isolated O\&G platforms or country-wide grids.

\section{REFERENCES}

[1] Norsk Petroleum Emissions to Air. Norwegianpetroleum.no. Feb. 17, 2020. URL: https://www. norskpetroleum.no/en/environment - andtechnology/emissions-to-air/ (visited on 02/17/2020).

[2] Kraft fra land til norsk sokkel. Technical Report. Norway: Oljedirektoratet, Norges vassdrags og energidirektorat, Petroleumtilsynet, Statens forurensningstilsyn, Jan. 2008, p. 99. URL: https://www. npd.no/globalassets/1 - npd/publikasjoner/rapporter/kraft- fra-landrapport.pdf (visited on 04/21/2021).

[3] Hywind Tampen - Floating Wind Power Project - Equinor.Com. Hywind Tampen: the world's first renewable power for offshore oil and gas. Mar. 3, 2020. URL: https://www.equinor.com/en/what-wedo/hywind-tampen.html (visited on 04/19/2021).

[4] E. F. Alves, D. d. S. Mota, and E. Tedeschi. "Sizing of Hybrid Energy Storage Systems for Inertial and Primary Frequency Control". In: Unpublished (2021).

[5] L. Riboldi et al. "Innovative Hybrid Energy System for Stable Power and Heat Supply in Offshore Oil \& Gas Installation (HES-OFF): System Design and Grid Stability”. In: ed. by S. Pierucci et al. Vol. 48. 30 European Symposium on Computer Aided Process Engineering. Elsevier, Jan. 1, 2020, pp. 211-216. DOI: 10 . 1016/B978 - 0 - 12 823377-1.50036-7.

[6] E. F. Alves. Efantnu/Hybrid-Ess-Design: Review 1 Release. Zenodo, Mar. 12, 2021. DOI: 10.5281/zenodo.4601067. URL: https://zenodo. org/record/4601067\#.YHoHlegzaUk (visited on 04/16/2021).

[7] H. S. Song and K. Nam. "Dual Current Control Scheme for PWM Converter under Unbalanced Input Voltage Conditions". In: IEEE Transactions on Industrial Electronics 46.5 (Oct. 1999), pp. 953-959. ISSN: 1557-9948. DOI: 10.1109/41.793344.
[8] D. Siemaszko. "Positive and Negative Sequence Control for Power Converters under Weak Unbalanced Networks". In: Railway and Ship Propulsion 2012 Electrical Systems for Aircraft. Oct. 2012, pp. 1-6. DOI: 10.1109/ESARS.2012.6387479.

[9] J. B. Glasdam. Harmonics in Offshore Wind Power Plants: Application of Power Electronic Devices in Transmission Systems. Springer, Oct. 26, 2015. 219 pp. ISBN: 978-3-319-26476-9.

[10] P. Brogan. "The Stability of Multiple, High Power, Active Front End Voltage Sourced Converters When Connected to Wind Farm Collector Systems". In: EPE Wind Energy Chapter Seminar. Stafford, 2010.

[11] P. B. Brogan et al. "Improved Converter Network Bridge Controller". U.S. pat. 20210021209A1. Jan. 21, 2021. URL: https://uspto.report/ patent/app/20210021209 (visited on 04/16/2021).

[12] D. Siemaszko. "Double Frame Control and Power Compensation for Power Converters Connected to Weak Networks with Disturbances". In: 2013 15th European Conference on Power Electronics and Applications (EPE). Sept. 2013, pp. 1-10. DOI: 10.1109/EPE.2013. 6631736.

[13] D. Siemaszko and A. C. Rufer. "Power Compensation Approach and Double Frame Control for Grid Connected Converters". In: 2013 IEEE 10th International Conference on Power Electronics and Drive Systems (PEDS). Apr. 2013, pp. 1263-1268. DOI: 10.1109/PEDS. 2013.6527213.

[14] F. Blaabjerg et al. "Overview of Control and Grid Synchronization for Distributed Power Generation Systems". In: IEEE Transactions on Industrial Electronics 53.5 (Oct. 2006), pp. 1398-1409. ISSN: 15579948. DOI: $10.1109 /$ TIE.2006.881997.

[15] C. J. O'Rourke et al. "A Geometric Interpretation of Reference Frames and Transformations: Dq0, Clarke, and Park". In: IEEE Transactions on Energy Conversion 34.4 (Dec. 2019), pp. 2070-2083. ISSN: 1558-0059. DOI: 10.1109/TEC.2019.2941175.

[16] F. Fröhr and F. Orttenburger. Introduction to Electronic Control Engineering. Berlin and München, Germany; London, UK: Siemens Aktiengesellschaft; Heyden \& Son LTD., 1982. ISBN: 0-85501-290-0.

[17] J. A. Suul et al. "Tuning of Control Loops for Grid Connected Voltage Source Converters". In: 2008 IEEE 2nd International Power and Energy Conference. Dec. 2008, pp. 797-802. DOI: 10.1109/ PECON.2008.4762584.

[18] L. H. Kocewiak. Harmonics in Large Offshore Wind Farms. Department of Energy Technology, Aalborg University, Denmark, 2012. ISBN: 978-87-92846-04-4.

[19] Z. Ye and H. Mohamadian. "Application of Modern Control Theory on Performance Analysis of Generalized Notch Filters". In: 2016 5th International Conference on Modern Circuits and Systems Technologies (MOCAST). May 2016, pp. 1-4. DOI: 10.1109/MOCAST.2016. 7495100.

[20] P. Rodriguez et al. "Decoupled Double Synchronous Reference Frame PLL for Power Converters Control". In: IEEE Transactions on Power Electronics 22.2 (Mar. 2007), pp. 584-592. ISSN: 1941-0107. DOI: 10.1109/TPEL.2006.890000.

[21] GEAFOL Cast-Resin Transformers 100 to 16,000 kVA. Catalogue TV1-2013. Erlangen, Germany: Siemens AG, 2013.

[22] ACS880-77LC/-87LC/-87CC Wind Turbine Converters. System Description 3AXD50000022022 Rev C. ABB, Apr. 27, 2020. URL: https : / / search . abb . com / library / Download . aspx ? DocumentID = 3AXD50000022022 \& LanguageCode $=$ en $\&$ DocumentPartId $=1 \&$ Action=Launch (visited on 06/26/2020).

[23] M. Liserre, F. Blaabjerg, and A. Dell'Aquila. "Step-by-Step Design Procedure for a Grid-Connected Three-Phase PWM Voltage Source Converter". In: International Journal of Electronics 91.8 (Aug. 1, 2004), pp. 445-460. ISSN: 0020-7217. DOI: 10.1080/ 00207210412331306186

[24] R. Peña-Alzola et al. "Analysis of the Passive Damping Losses in LCL-Filter-Based Grid Converters". In: IEEE Transactions on Power Electronics 28.6 (June 2013), pp. 2642-2646. ISSN: 1941-0107. DOI: 10.1109/TPEL.2012.2222931.

[25] R. N. Beres et al. "A Review of Passive Power Filters for ThreePhase Grid-Connected Voltage-Source Converters". In: IEEE Journal of Emerging and Selected Topics in Power Electronics 4.1 (Mar. 2016), pp. 54-69. ISSN: 2168-6785. DOI: 10.1109/JESTPE. 2015. 2507203.

[26] H. Brantsæter et al. "Passive Filter Design and Offshore Wind Turbine Modelling for System Level Harmonic Studies". In: Energy Procedia 
80 (2015), pp. 401-410. ISSN: 1876-6102. DOI: 10.1016/j.egypro. 2015.11.444.

[27] The Top-Performing Wind Converter Trusted Technology for Maximum Power Yield - SINAMICS W180. Brochure PDLD-B10099-00-7600. Siemens AG, 2017. URL: https : / / assets . new . siemens . com / siemens / assets / api / uuid : 26ef63b7cae9657fae9e98618212ba874f3fd729 455_170680_ws_sinamicsw180us.pdf (visited on 09/27/2020).

\section{APPENDIX A}

\section{REFERENCE FRAMES AND TRANSFORMATIONS}

\section{A. Positive Sequence Rotating Reference Frame}

Equation (A.1) shows the matrix $\mathbf{T}_{+}$used in the $a b c \mapsto d q_{+}$ and $d q_{+} \mapsto a b c$ transformations. The power converters analyzed in this study are connected to the grid via an ungrounded delta-wye transformer. Therefore, the zero sequence is disregarded.

$$
\mathbf{T}_{+}=\left[\begin{array}{lll}
\sin (\omega t) & \sin (\omega t-2 \pi / 3) & \sin (\omega t+2 \pi / 3) \\
\cos (\omega t) & \cos (\omega t-2 \pi / 3) & \cos (\omega t+2 \pi / 3)
\end{array}\right]
$$

The $a b c \rightarrow d q_{+}$transformation is given by (A.2).

$$
\left[\begin{array}{l}
m_{d}(t) \\
m_{q}(t)
\end{array}\right]=\frac{2}{3} \mathbf{T}_{+}(t)\left[\begin{array}{l}
m_{a}(t) \\
m_{b}(t) \\
m_{c}(t)
\end{array}\right]
$$

The variables $m_{a b c}$ represent the instantaneous measurements of either phase to neutral voltages or line currents. The angular frequency $\omega$ in $\mathbf{T}_{+}$is adopted as the same angular frequency of the grid. Moreover, the initial phase of the grid voltage between phase $a$ and the neutral is adopted as zero, i.e., $v_{a}(t)=V \sin (\omega t)$.

The $d q_{+} \mapsto a b c$ transformation is given by (A.3), where the superscript $T$ stands for transpose.

$$
\left[\begin{array}{l}
m_{a}(t) \\
m_{b}(t) \\
m_{c}(t)
\end{array}\right]=\mathbf{T}_{+}^{T}(t)\left[\begin{array}{l}
m_{d}(t) \\
m_{q}(t)
\end{array}\right] .
$$

\section{B. Negative Sequence Rotating Reference Frame}

A measurement $\left[\begin{array}{lll}m_{a} & m_{b} & m_{c}\end{array}\right]^{T}$ can be moved to the negative sequence RRF if the mid and right columns of $\mathbf{T}_{+}$are swapped. The result is shown in (A.4). This RRF is denoted by $d q_{-}$in this paper. For the $a b c \mapsto d q_{-}$and $d q_{-} \mapsto a b c$ transformations, replace $\mathbf{T}_{+}$by $\mathbf{T}_{-}$in (A.2) and (A.3).

$$
\mathbf{T}_{-}=\mathbf{T}_{+}\left[\begin{array}{lll}
1 & 0 & 0 \\
0 & 0 & 1 \\
0 & 1 & 0
\end{array}\right]
$$

\section{Negative $\omega$ Rotating Reference Frame}

A measurement $\left[\begin{array}{lll}m_{a} & m_{b} & m_{c}\end{array}\right]^{T}$ can be moved to the negative $\omega$ RRF if the sign of the angular speed in $\mathbf{T}_{+}$is reversed. The result is shown in (A.5). This RRF is denoted by $d q_{-\omega}$ in this paper. For the $a b c \mapsto d q_{-\omega}$ and $d q_{-\omega} \mapsto a b c$ transformations, replace $\mathbf{T}_{+}$by $\mathbf{T}_{-\omega}$ in (A.2) and (A.3).

$$
\mathbf{T}_{-\omega}=\left[\begin{array}{cc}
-1 & 0 \\
0 & 1
\end{array}\right] \mathbf{T}_{+}\left[\begin{array}{lll}
1 & 0 & 0 \\
0 & 0 & 1 \\
0 & 1 & 0
\end{array}\right]=\left[\begin{array}{cc}
-1 & 0 \\
0 & 1
\end{array}\right] \mathbf{T}_{-}
$$

\section{APPENDIX B \\ Case Study Data}

TABLE B.I: Case study platform.

\begin{tabular}{lcc}
\hline Parameter & & Value \\
\hline System rated frequency & $f_{n}$ & $60 \mathrm{~Hz}$ \\
Generator rated apparent power & & $44 \mathrm{MVA}$ \\
Gas turbine rated power & & $35 \mathrm{MW}$ \\
Gen. and busbar rated ac voltage & & $11 \mathrm{kV}$ \\
ESS rated apparent power & $S_{b}$ & $9.65 \mathrm{MVA}$ \\
ESS HV ac rated voltage & & $11 \mathrm{kV}$ \\
ESS LV ac rated voltage & & $690 \mathrm{~V}$ \\
ESS LV ac rated line current & $I_{b}$ & $8075 \mathrm{~A}$ \\
ESS LV base impedance & $Z_{b}$ & $0.049337 \Omega$ \\
LSC switching frequency & $f_{s w}$ & $2.5 \mathrm{kHz}-4 \mathrm{kHz}$ \\
LSC dc voltage & $U_{d c}$ & $1200 \mathrm{~V}$ \\
\hline
\end{tabular}

TABLE B.II: Estimating a representative $X / R$.

\begin{tabular}{lccc} 
& \multicolumn{3}{c}{ Value at (base) in pu } \\
\hline Parameter & & $(88 \mathrm{MVA})$ & $(9.65 \mathrm{MVA})$ \\
\hline Transient reactance & $x_{d}^{\prime}$ & 0.299 & 0.032788 \\
Stator resistance & $r_{s}$ & 0.0242 & 0.002654 \\
Breaker+HV devices res. & $r_{b}$ & 0.007273 & 0.000798 \\
Load resistance & $r_{L}$ & 1.964 & 0.215402 \\
Transformer reactance & $x_{t}$ & - & 0.060 \\
Transformer resistance & $r_{t}$ & - & 0.005 \\
Capacitor impedance & $x_{c}$ & - & 20 \\
Damping resistor & $r_{c}$ & - & 0.340 to 0.328 \\
Main reactor resistance & $r_{r}$ & - & 0.01 \\
\hline
\end{tabular}

